\title{
Broadband extraction of tissue optical properties using a portable hybrid time-resolved continuous wave instrumentation: characterization of ex vivo organs
}

\author{
Marcelo Saito Nogueira ${ }^{*, \Delta, 1,2}$, Michele Lacerenza ${ }^{3, \Lambda}$, Sanathana Konugolu Venkata Sekar', Mauro \\ Buttafava ${ }^{4}$, Antonio Pifferi ${ }^{3,5}$, Alberto Tosi ${ }^{4}$, Davide Contini ${ }^{1}$, Stefan Andersson-Engels ${ }^{1,2}$ \\ ${ }^{I}$ Tyndall National Institute, Cork, Ireland, Lee Maltings Complex, Dyke Parade, Cork, Ireland, Postcode: T12R5CP \\ ${ }^{2}$ Department of Physics, University College Cork, College Road, University College, Cork, Postcode: T12K8AF \\ ${ }^{3}$ Dipartimento di Fisica, Politecnico di Milano, Piazza Leonardo da Vinci 32, 20133 Milan, Italy \\ ${ }^{4}$ Dipartimento di Elettronica, Informazione e Bioingegneria, Politecnico di Milano, Italy \\ ${ }^{5}$ Instituto di Fotonica e Nanotecnologie, Consiglio Nazionale delle Ricerche, 20133 Milano, Italy \\ ${ }^{4}$ Equally contributing authors \\ *marcelosaitonogueira@gmail.com
}

\begin{abstract}
Successful demonstration of a unique portable CW-TDDOS system for accurate and multiwavelength retrieval of tissue optical properties. Determining these properties has potential to improve the diagnosis and treatment outcomes in clinical and sports settings.
\end{abstract}

\section{Introduction}

Current broadband continuous wave $(\mathrm{CW})$ systems are able to perform reflectance and transmittance measurements, which can be used to extract tissue optical properties. The extended wavelength range to the near-infrared allows the investigation of the optical properties in the wavelength range where light penetrates deeper into tissues (optical windows). Characterizing tissue optical properties in the optical windows is attractive to the medical device industry, as they have been exploited for novel optical diagnostics tools and treatment modalities requiring information from centimeters under the tissue surface. Optical properties can be extracted by using analytical models or numerical methods based on the radiative transfer equation. The accuracy for broadband CW systems relies on the constraints applied by the wavelength-dependency of the chromophore spectra and scattering properties. On the other hand, the amplitude of the absorption and scattering coefficients may differ depending on the model used to describe the CW signals. The possible inaccuracy in the amplitude of the optical properties can be overcome by calibrating the optical properties with a gold standard technique such as time-domain diffuse optical spectroscopy (TDDOS) systems. With this in mind, we present a novel portable combination between CW and TDDOS for accurate estimation of broadband optical properties in tissue-mimicking phantoms and ex vivo organs. The system will be used in a colorectal cancer detection study currently approved by the research ethics committee.

\section{Material and Methods}

The portable system consists of a broadband CW diffuse transmittance system and a near-infrared TDDOS system used independently in order to prevent crosstalk between the two instruments. The CW system [1] contains a broadband light source (HL-2000, Ocean Optics, Edinburgh, United Kingdom) which sends the excitation light to the sample through a 1000- $\mu$ m-core 0.39 NA Low-OH-Silica fiber optic probe (M35L01 Fiber Patch Cable, Thorlabs, Munich, Germany). The transmitted light is detected by a visible wavelength spectrometer (QE-Pro, Ocean Optics, Edinburgh, United Kingdom). After collecting intensity measurements, the data was post-processed in order to obtain the sample optical properties. The TDDOS system, developed at Politecnico di Milano, employs two pulsed laser diodes $(670 \mathrm{~nm}$ and $830 \mathrm{~nm}$ ) and a SIPM detector to estimate the absolute optical properties (absorption and reduced scattering coefficients, $\mu_{\mathrm{a}}$ and $\mu_{\mathrm{s}}$ ' respectively) of the tissue under study [2,3]. The portability of the two systems is shown in figure 1A.

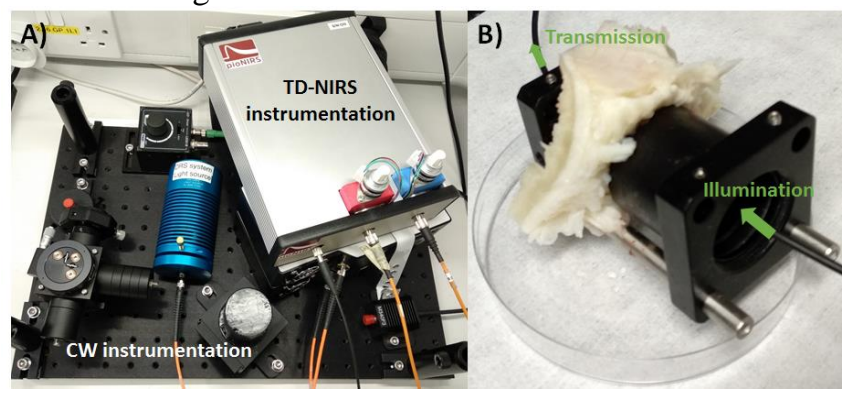

Fig.1 A) Portable broadband CW and TDDOS instrumentation and B) Transmittance measurement setup. 
The system was characterized by using a phantom matrix kit [4] in order to check the performance of the combined (CW+TD) portable system. The phantom matrix contains 24 phantoms with $\mu_{\mathrm{a}}$ ranging from 0.1 to $1 \mathrm{~cm}^{-1}$ and $\mu_{\mathrm{s}}$ ' from 5 to $25 \mathrm{~cm}^{-1}$ and well characterized using broadband time domain system [5]. Then, the optical properties of $e x$ vivo tissues the liver, bone marrow, heart, kidney, fat, and muscle were extracted by using our hybrid system. The transmission measurements were performed by using a customized probe or the setup shown in figure 1B.

\section{Results}

The linearity of the portable system at $825 \mathrm{~nm}$ to extract optical properties $\left(\mu_{\mathrm{a}}\right.$ and $\left.\mu_{\mathrm{s}}{ }^{\prime}\right)$ of the phantom matrix kit is shown in Fig. 2.
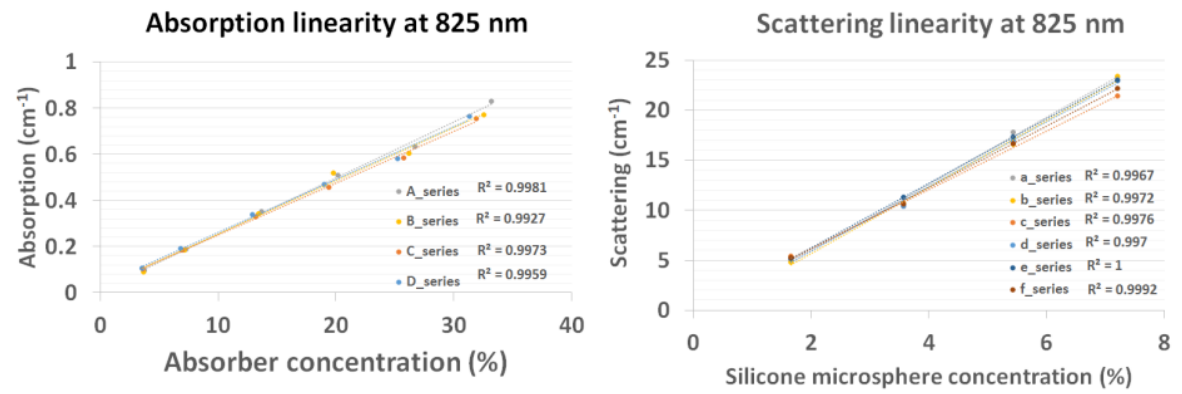

Fig.2 TDDOS characterization of the absorption (left) and scattering (right) linearity of the phantom matrix kit.

The estimation of the tissue optical properties is consistent to what is biologically expected, as the $\mu_{\mathrm{a}}$ of high vascularized tissues (e.g. muscle, heart and kidney) is from 2 to 8 times higher compared to relatively low vascularized ones (e.g. bone marrow), due to the differences in blood, lipid and water content. Our results suggest our hybrid CW-TDDOS system was capable of accurate extraction of broadband tissue optical properties.
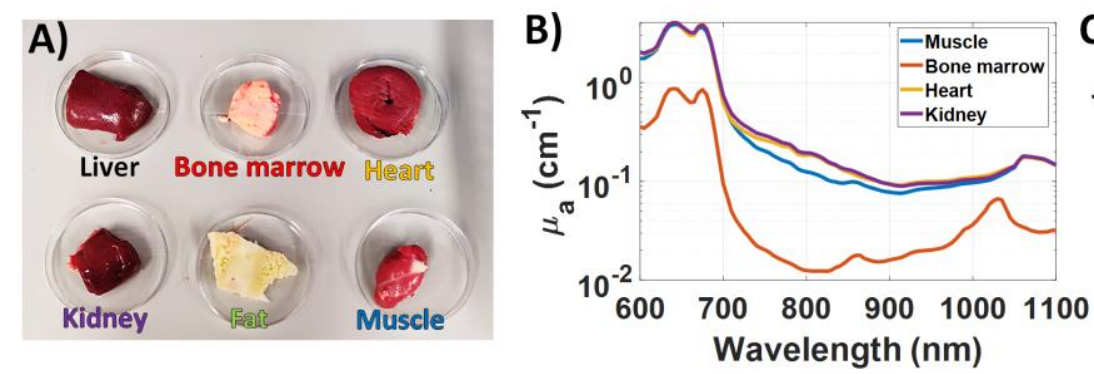

Fig. 3 A) Measured ex vivo tissues, $B$ ) $\mu_{\mathrm{a}}$ and $C$ ) $\mu_{\mathrm{s}}$ ' of the ex vivo tissues.



\section{Conclusions}

We successfully demonstrate the accuracy of a unique portable CW-TDDOS system for multiwavelength retrieval of tissue optical properties, which can be used for clinical or sports medicine applications. Moreover, our hybrid CW-TDDOS has potential to extract the biochemical depth profile of biological tissues in order to improve the diagnosis and treatment outcomes. Future work includes in vivo colorectal cancer detection studies.

\section{References}

[1] Nogueira MS et al., "Diffuse reflectance spectroscopy for determination of optical properties and chromophore concentrations of mice internal organs in the range of $350 \mathrm{~nm}$ to $1860 \mathrm{~nm}$ ”. Biophotonics: Photonic Solutions for Better Health Care VI 2018 May 17 (Vol. 10685 , p. 106853G).

[2] M. Lacerenza et al., "A wearable time-domain near-infrared spectroscopy system” Proc. SPIE 11074, Diffuse Optical Spectroscopy and Imaging VII, 1107404 (11 July 2019).

[3] M. Buttafava et al., "A Compact Two-Wavelength Time-Domain NIRS System Based on SiPM and Pulsed Diode Lasers," IEEE Photonics J., vol. 9, no. 1, 2017.

[4] S. Konugolu Venkata Sekar et al., "Solid phantom recipe for diffuse optics in biophotonics applications : a step towards anatomically correct 3D tissue phantoms," vol. 10, no. 4, pp. 2090-2100, 2019.

[5] S. Konugolu Venkata Sekar et al., "Broadband (600-1350 nm) time-resolved diffuse optical spectrometer for clinical use," IEEE J. Sel. Top. Quantum Electron. 22(3), 7100609 (2016). 